\title{
CROSSING NUMBER OF LINKS FORMED BY EDGES OF A TRIANGULATION
}

\author{
SIMON A. KING
}

\begin{abstract}
We study the crossing number of links that are formed by edges of a triangulation $\mathcal{T}$ of $S^{3}$ with $n$ tetrahedra. We show that the crossing number is bounded from above by an exponential function of $n^{2}$. In general, this bound can not be replaced by a subexponential bound. However, if $\mathcal{T}$ is polytopal (resp. shellable) then there is a quadratic (resp. biquadratic) upper bound in $n$ for the crossing number. In our proof, we use a numerical invariant $p(\mathcal{T})$, called polytopality, that we have introduced in 5 .

Primary: 57M25, 57Q15

Secondary: 52C45, 52B22

Keywords: crossing number, triangulation, polytopality
\end{abstract}

\section{INTRODUCTION}

The aim of this paper is to study properties of knots and links that are formed by edges of a triangulation of the 3-dimensional sphere $S^{3}$. For any $n \in \mathbb{N}$, let $\mathbb{T}_{n}$ denote the set of triangulations of $S^{3}$ with $n$ tetrahedra. The number of simplicial complexes with $n$ tetrahedra is finite, and the number of simple edge paths in a simplicial complex is finite as well. Therefore, there are only finitely many equivalence classes of links in $S^{3}$ occuring in the 1 -skeleton $\mathcal{T}^{1}$ of some $\mathcal{T} \in \mathbb{T}_{n}$. Thus, if $f(\cdot)$ is any numerical link invariant (e.g., bridge number, crossing number, $k$-th coefficient of the Jones polynomial) then

$$
F(n)=\sup _{\mathcal{T} \in \mathbb{T}_{n}, L \subset \mathcal{T}^{1}} f(L)
$$

is a finite number for all $n \in \mathbb{N}$.

In 四 and [⿹𠃌丨丿 bridge number of $L$. However, there are infinitely many non-equivalent links whose bridge number is smaller than $F(n)$, for instance, two-bridge knots. The aim of this paper is to prove an upper bound for $F(n)$ when $f(L)$ is the crossing number of $L$. Such an estimate is substantially stronger than an estimate for the bridge number, in the sense that there are only finitely many equivalence classes of links with a crossing number smaller than $F(n)$.

We recall the notions of crossing number and bridge number of a link $L \subset S^{3}$. The crossing number $C r(L)$ of $L$ is the minimal number of crossings in a link diagram representing $L$. This is a natural measure of complexity of links, and was already used in the knot tables of Tait and Little in 1900. The bridge number has been introduced by H. Schubert [8] in 1954. It can be defined as follows. Let $I=[0,1]$ denote the unit interval. Let $H: S^{2} \times I \rightarrow S^{3}$ be a smooth embedding with $L \subset H\left(S^{2} \times I\right)$. Let $\pi_{I}: S^{2} \times I \rightarrow I$ denote the projection to the second factor. On gets a map $h: L \rightarrow S^{2}$ by $h(p)=\pi_{I} \circ H^{-1}(p)$, for $p \in L$. We call $p \in L$ a critical point of $H$ with respect to $L$ if $H\left(S^{2} \times\{h(p)\}\right)$ is not transversal to $L$ in $p$. Thus, generically a critical point is an isolated local maximum or minimum of $h$. We denote the number of critical points by $c(H, L)$. Now, the bridge number of $L$ is

$$
b(L)=\frac{1}{2} \min _{H} c(H, L),
$$

where the minimum is taken over all embeddings $H: S^{2} \times I \rightarrow S^{3}$ with $L \subset H\left(S^{2} \times I\right)$. The factor $\frac{1}{2}$ is needed to make this definition consistent with Schubert's original definition [8]. 
Link invariants also arise in the study of polytopal and shellable triangulations of $S^{3}$, as explained in the next paragraphs. Recall that a triangulation of the $d$-dimensional sphere $S^{d}$ is polytopal, if it is isomorphic to the boundary complex of a convex $(d+1)$-polytope. A triangulation of $S^{d}$ is shellable, if there is an order $\sigma_{1}, \ldots, \sigma_{n}$ of its $d$-simplices so that $\bigcup_{i=1}^{k} \overline{\sigma_{i}}$ is a $d$-dimensional closed ball, for $k=1, \ldots, n-1$. For a more general definition of shellable cellular complexes, see [9]. Any polytopal triangulation is shellable [2]. There are shellable triangulations of $S^{3}$ that are not polytopal, and there are triangulations of $S^{3}$ that are not shellable [9].

Let $\mathcal{T} \in \mathbb{T}_{n}$ be a triangulation of $S^{3}$ with $n$ tetrahedra, and let $K \subset \mathcal{T}^{1}$ be a knot formed by $k$ edges of $\mathcal{T}$. It follows from the work of Lickorish [6] that $b(K) \leq k$ under the assumption that $\mathcal{T}$ is shellable. Armentrout [1] obtained $b(K) \leq \frac{1}{2} k$ under the assumption that the dual of $\mathcal{T}$ is shellable. Recently Ehrenborg and Hachimori [3] obtained the sharp bounds $b(K) \leq \frac{1}{2} k$ if $T$ is shellable and $b(K) \leq \frac{1}{3} k$ if $\mathcal{T}$ is vertex decomposable (we will not define this notion here). In the same way, one can show that if $L \subset \mathcal{T}^{1}$ is a link (but not necessarily a knot) then $b(L)$ is bounded from above by a linear function of $n$, provided $\mathcal{T}$ satisfies one of the mentioned assumptions. These assumptions are in fact very strong, since in general there is no subexponential upper bound for $b(L)$ in terms of $n$, see $\mid 5$. Without any geometric assumption on $\mathcal{T}$, we found $b(L)<2^{190 n^{2}}$ by a complexity analysis of the Rubinstein-Thompson algorithm for the recognition of $S^{3}$, see [4].

By the following main theorem of this paper, the crossing number of a link in $\mathcal{T}^{1}$ is also sensitive for geometric properties of $\mathcal{T}$.

Theorem 1. Let $\mathcal{T}$ be a triangulation of $S^{3}$ with $n$ tetrahedra, and let $L \subset \mathcal{T}^{1}$ be a link.

1. If $\mathcal{T}$ is polytopal then $\operatorname{Cr}(L)<4 n^{2}$.

2. If $\mathcal{T}$ or its dual cellular decomposition is shellable then $\operatorname{Cr}(L)<10^{9} n^{4}$.

3. In general, $\operatorname{Cr}(L)<2^{810 n^{2}}$.

So far as known to the author, these are the first upper bounds for $\operatorname{Cr}(L)$ in terms of $\mathcal{T}$. Certainly these bounds are not optimal, we did not try to prove sharp bounds. However, since $\operatorname{Cr}(L) \geq b(L)$, our results in [5] imply that the general bound in the third part of Theorem 1 can not be replaced by a subexponential bound. Thus, the assumptions in the first two parts of Theorem 1 can not be removed.

We outline the proof of Theorem 1. Let $\mathcal{T} \in \mathbb{T}_{n}$ and let $L \subset \mathcal{T}^{1}$. If $\mathcal{T}$ is polytopal, then one can isotope its 2-skeleton in $\mathbb{R}^{3} \subset S^{3}$ so that all edges become straight line segments. Thus, an orthogonal projection yields a link diagram for $L$ with at most one crossing for each pair of edges. This yields the first part of Theorem 11.

The idea for the proof of the second and third part of Theorem 1 is to transform the triangulation $\mathcal{T}$ into a polytopal triangulation $\tilde{\mathcal{T}}$ by a finite number of local changes (e.g., stellar subdivisions), so that $\tilde{\mathcal{T}}^{1}$ contains a copy $\tilde{L}$ of $L$, where the number of edges forming $\tilde{L}$ is controlled in terms of the number of local changes. This reduces the problem to the first part of Theorem 1. For this purpose, we use the notion of polytopality that we have introduced in [5]. The polytopality $p(\mathcal{T})$ is a numerical invariant of a triangulation $\mathcal{T}$ of $S^{3}$. Its definition is inspired by the bridge number of links, see Section 2. In [5], we have shown how $\tilde{\mathcal{T}}$ can be constructed from $\mathcal{T}$, so that the number of local changes is bounded from above by a quadratic function of $p(\mathcal{T})$. Since $\tilde{\mathcal{T}}$ is polytopal, we obtain a biquadratic upper bound for $\operatorname{Cr}(\tilde{L})=\operatorname{Cr}(L)$ in terms of $p(\mathcal{T})$. We have shown [5] that $p(\mathcal{T}) \leq 7 n$ if $\mathcal{T}$ or its dual is shellable, and $p(\mathcal{T})<2^{200 n^{2}}$ in general. This yields the estimates claimed in the second and third part of Theorem $\mathbb{1}$.

The rest of this paper is organized as follows. In Section 2 we define the polytopality $p(\mathcal{T})$ of a triangulation $\mathcal{T}$ of $S^{3}$ and recall from [5] how to construct from $\mathcal{T}$ a polytopal triangulation by a series of local changes whose length is bounded in terms of $p(\mathcal{T})$. In Section 3, we start with a proof of the first part of Theorem 1. Then, we formulate and prove a bound for the crossing number of a link $L \subset \mathcal{T}^{1}$ in terms of $p(\mathcal{T})$. This bound 
together with estimates for $p(\mathcal{T})$ from [5] immediately yields the second and third part of Theorem 1 .

\section{Polytopality of triangulations}

Let $\mathcal{T}$ be a triangulation of $S^{3}$. The aim of this section is to expose a numerical invariant $p(\mathcal{T})$, called polytopality. Although its definition is purely topological, $p(\mathcal{T})$ turns out to be a measure for the geometric complexity of $\mathcal{T}$.

We recall the definition of $p(\mathcal{T})$ from $[5]$. Let $\mathcal{C}$ be the dual cellular decomposition of $\mathcal{T}$, with 1-skeleton $\mathcal{C}^{1}$. Let $H: S^{2} \times I \rightarrow S^{3}$ be a smooth embedding with $\mathcal{C}^{1} \subset H\left(S^{2} \times I\right)$. Let $\pi_{I}: S^{2} \times I \rightarrow I$ denote the projection to the second factor. This gives rise to a map $h: \mathcal{C}^{1} \rightarrow S^{2}$ by $h(p)=\pi_{I} \circ H^{-1}(p)$, for $p \in \mathcal{C}^{1}$. We call $p \in \mathcal{C}^{1}$ a critical point of $H$ with respect to $\mathcal{C}^{1}$ if $H\left(S^{2} \times\{h(p)\}\right)$ is not transversal to $\mathcal{C}^{1}$ in $p$. Thus, generically a critical point is a vertex of $\mathcal{C}^{1}$ or an isolated local maximum or minimum of $h$ in the interior of an edge of $\mathcal{C}$. We denote the number of critical points of $H$ with respect to $\mathcal{C}^{1}$ by $c\left(H, \mathcal{C}^{1}\right)$ and define

$$
p(\mathcal{T})=\min _{H} c\left(H, \mathcal{C}^{1}\right),
$$

the polytopality of $\mathcal{T}$, where the minimum is taken over all embeddings $H: S^{2} \times I \rightarrow S^{3}$ with $\mathcal{C}^{1} \subset H\left(S^{2} \times I\right)$.

The definition of polytopality is very similar to the definition of bridge number in Section 1 . One can think of $p(\mathcal{T})$ as a bridge number of the spatial graph $\mathcal{C}^{1} \subset S^{3}$. We remark that already the abstract graph $\mathcal{C}^{1}$ contains much information about $\mathcal{T}$. For instance, if $\mathcal{T}$ is polytopal than it is already determined by the abstract graph $\mathcal{C}^{1}$, see [9]. In [0], we obtained the following estimates.

Theorem 2 (see [5]). Let $\mathcal{T} \in \mathbb{T}_{n}$.

1. If $\mathcal{T}$ is polytopal then $p(\mathcal{T})=n$.

2. If $\mathcal{T}$ or its dual cellular decomposition is shellable then $p(\mathcal{T}) \leq 7 n$.

3. In general, $n \leq p(\mathcal{T})<2^{200 n^{2}}$. The upper bound can not be replaced by a subexponential bound.

It is not known to the author whether there is a non-polytopal triangulation $\mathcal{T} \in \mathbb{T}_{n}$ with $p(\mathcal{T})=n$. Theorem 2 shows that $p(\mathcal{T})$ is sensitive for geometric properties of $\mathcal{T}$. However, the connection to geometry is even stronger, since $p(\mathcal{T})$ measures to what extend $\mathcal{T}$ fails to be polytopal, as explained in the rest of this section.

Definition 1. Let $\mathcal{T}_{1}$ and $\mathcal{T}_{2}$ be triangulations of $S^{3}$, and let $e$ be an edge of $\mathcal{T}_{2}$ with $\partial e=\{a, b\}$. Suppose that $\mathcal{T}_{1}$ is obtained from $\mathcal{T}_{2}$ by removing the open star of $e$ and identifying the join $a * \sigma$ with $b * \sigma$ for any simplex $\sigma$ in the link of $e$. Then $\mathcal{T}_{2}$ is the result of an expansion of $\mathcal{T}_{1}$ along $e$.

Since an expansion increases the number of vertices by one and the number of simplicial complexes with a given number of vertices is finite, it is easy to see that the number of possible expansions of $\mathcal{T}_{1}$ is finite up to isotopy. Any stellar subdivision of a simplex of $\mathcal{T}$ is an expansion.

It is a consequence of the "Hauptvermutung" for 3-dimensional manifolds [7] that any triangulation $\mathcal{T}$ of $S^{3}$ can be turned into a polytopal triangulation by a series of expansions. We define $d(\mathcal{T})$ as the length of a shortest series of expansions that transforms $\mathcal{T}$ into a polytopal triangulation. Our next theorem shows that $d(\mathcal{T})$, defined in terms of discrete geometry, is closely related to $p(\mathcal{T})$, defined in terms of topology.

Theorem 3 (see [5]). For any $\mathcal{T} \in \mathbb{T}_{n}$ holds

$$
\frac{p(\mathcal{T})}{3 n}-\frac{5}{3}-n<d(\mathcal{T}) \leq 512(p(\mathcal{T}))^{2}+869 p(\mathcal{T})+376
$$




\section{Proof of Theorem 1}

Proof of the first part of Theorem $\mathbf{\text { }}$. Let $\mathcal{T} \in \mathbb{T}_{n}$ be a polytopal triangulation of $S^{3}$ with $n$ tetrahedra and let $L \subset \mathcal{T}^{1}$ be a link formed by $k$ edges. Since $\mathcal{T}$ has at most $2 n$ edges, it suffices to prove $C r(L)<k^{2}$. Since $\mathcal{T}$ is polytopal, it has a so-called $S$ chlegel diagram [9], i.e., the simplicial complex obtained from $\mathcal{T}$ by removing a single tetrahedron can be embedded into $\mathbb{R}^{3}$ so that any simplex is euclidian. In particular, the link $L \subset \mathcal{T}^{1}$ is a union of $k$ straight line segments. It is possible to obtain a link diagram for $L$ by an orthogonal projection onto some plane. Since an orthogonal projection yields at most one crossing for any pair of edges, we obtain $C r(L)<k^{2}$.

In order to prove the second and third part of Theorem 1 1 , we formulate and prove the following estimate for $\operatorname{Cr}(L)$ in terms of $p(\mathcal{T})$.

Theorem 4. Let $\mathcal{T}$ be a triangulation of $S^{3}$, and let $L \subset \mathcal{T}^{1}$ be a link formed by $k$ edges of $\mathcal{T}$. Then

$$
C r(L)<\left(k+512(p(\mathcal{T}))^{2}+869 p(\mathcal{T})+376\right)^{2} .
$$

Proof. By Theorem 3, there is a polytopal triangulation $\tilde{\mathcal{T}}$ of $S^{3}$, obtained from $\mathcal{T}$ be a series of at most $512(p(\mathcal{T}))^{2}+869 p(\mathcal{T})+376$ expansions. If a triangulation of $S^{3}$ is obtained from $\mathcal{T}$ by a single expansion, then its 1 -skeleton contains a copy of $L$ formed by at most $k+1$ edges. Thus, by induction on the number of expansions, there is a link $\tilde{L} \subset \tilde{\mathcal{T}}^{1}$ equivalent to $L$, formed by at most $k+512(p(\mathcal{T}))^{2}+869 p(\mathcal{T})+376$ edges. Since $\tilde{\mathcal{T}}$ is polytopal, we obtain $\operatorname{Cr}(L)=C r(\tilde{L})<\left(k+512(p(\mathcal{T}))^{2}+869 p(\mathcal{T})+376\right)^{2}$ as in the proof of the first part of Theorem 1 .

Proof of the second and third part of Theorem 1 . Let $\mathcal{T} \in \mathbb{T}_{n}$, and let $L \subset \mathcal{T}^{1}$ be a link. Since $L$ is formed by at most $2 n$ edges, Theorems 2 and 4 immediately yield

$$
\begin{aligned}
C r(L) & <\left(25088 n^{2}+6085 n+376\right)^{2} \\
& <10^{9} n^{4}
\end{aligned}
$$

provided $\mathcal{T}$ is shellable, and in general

$$
\begin{aligned}
C r(L) & <\left(512 \cdot 2^{400 n^{2}}+869 \cdot 2^{200 n^{2}}+2 n+376\right)^{2} \\
& <2^{810 n^{2}} .
\end{aligned}
$$

\section{REFERENCES}

[1] Armentrout, S.: Knots and shellable cell partitionings of $S^{3}$. Illinois J. Math. 38, 347-365, (1994).

[2] Bruggesser, H. and Mani, P.: Shellable decompositions of cells and spheres. Math. Scand 29, 197-205 (1971).

[3] Ehrenborg, R. and Hachimori, M.: Non-constructible complexes and the bridge index. Preprint 2000.

[4] King, S.: The size of triangulations supporting a given link. Geometry \& Topology 5, 369-398 (2001). See [0] for the slightly better bound that we use here.

[5] King, S.: Polytopality of triangulations. PhD thesis,

http://www-irma.u-strasbg.fr/irma/publications/2001/01017.shtml.

[6] Lickorish, W. B. R.: Unshellable triangulations of spheres. European J. Combinatorics 12, 527-530 (1991).

[7] Moise, E. E.: Affine structures in 3-manifolds. Ann. Math. 56, 96-114 (1952).

[8] Schubert, H.: Über eine numerische Knoteninvariante. Math. Z. 61, 245-288 (1954).

[9] Ziegler, G.: Lectures on polytopes, Graduate Texts in Mathematics 152, (Springer Verlag 1995).

Simon A. King, Department of Mathematics, Darmstadt University of Technology, SchlossGartenstr. 7, 64289 Darmstadt, Germany

E-mail address: king@mathematik.tu-darmstadt.de 